\section{Perforated peptic ulcer imaged directly by computed tomography}

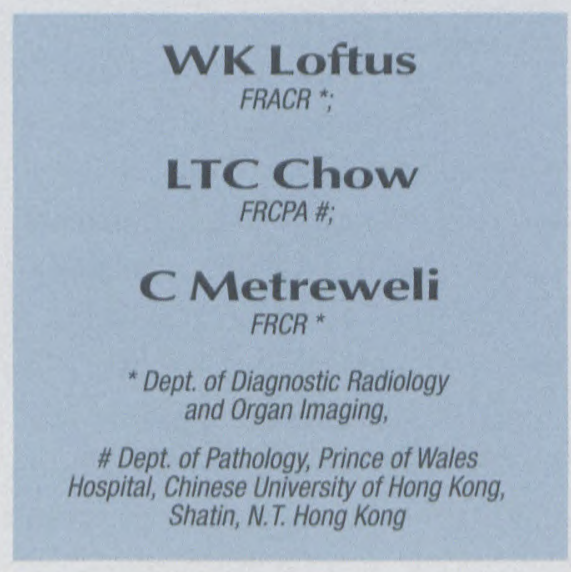

\section{Introduction}

Perforation is said to occur in $5-10 \%$ of the overall population who have peptic ulcer (PU). ${ }^{1}$ Recurrent ulceration following surgery for $\mathrm{PU}$ is well recognized and in $95 \%$ is secondary to surgery for duodenal rather than gastric ulcer. It is seen in about $3 \%$ of cases. Recurrent ulceration usually occurs following a gastrojejunal anastomosis but can develop even after vagotomy alone. Typically, however, these ulcers develop at an anastomotic site and perforation of these recurrent ulcers occurs in about $10 \%{ }^{2}$ While CT has no role in uncomplicated PU, it is useful in detecting perforation or penetration. The principal CT signs of perforation are extraluminal gas or, when administered, extravasated oral contrast. The location of a focal gas or contrast collection indicates a perforation in that area although the actual perforation itself is unlikely to be seen, ${ }^{1}$ even when contrast is used. ${ }^{3}$ The direct demonstration of an actual perforation does not seem to have been previously reported. We present a case in which the perforation was imaged by CT.

\section{Case report}

A 79 year old man presented with sudden onset of severe abdominal pain for 12 hours. Relevant past medical history included a Billroth type II gastrectomy for PU more than 10 years before. Clinically the patient had an acute abdomen. There was an elevated serum amylase and the clinical differential diagnosis was between pancreatitis and a perforated peptic ulcer. Plain films were unremarkable and did not reveal free gas. US showed free fluid in the abdomen consistent with either diagnosis. The gallbladder was normal with no gallstones but as the pancreas could

by CT. 


\section{Perforated peptic ulcer imaged directly by computed tomography}

\section{from page 31}

not be visualised, due to bowel gas, the patient was referred for CT. This demonstrated intraperitoneal free gas and fluid (Figure 1). There was a focal

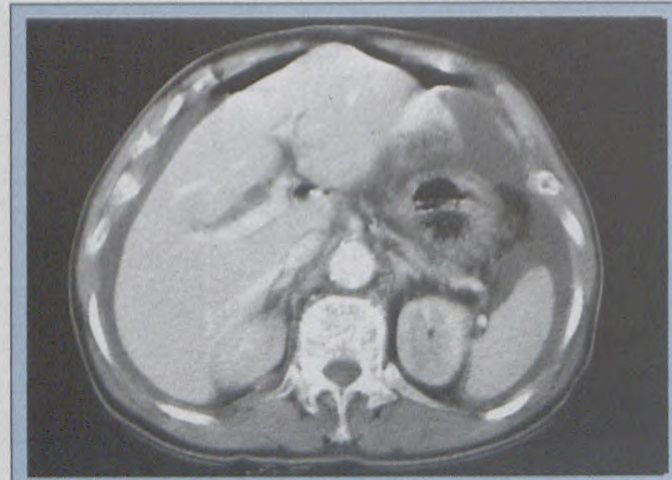

Figure 1: Axial CT image shows free intraperitoneal gas anteriorly and in the porta and extensive free fluid. Note the nasogastric tube in the stomach remnant.

defect in the posterior wall of the gastric remnant consistent with a perforated PU (Figures 2 and 3). The patient refused surgery and died within 24 hours. Post mortem confirmed the

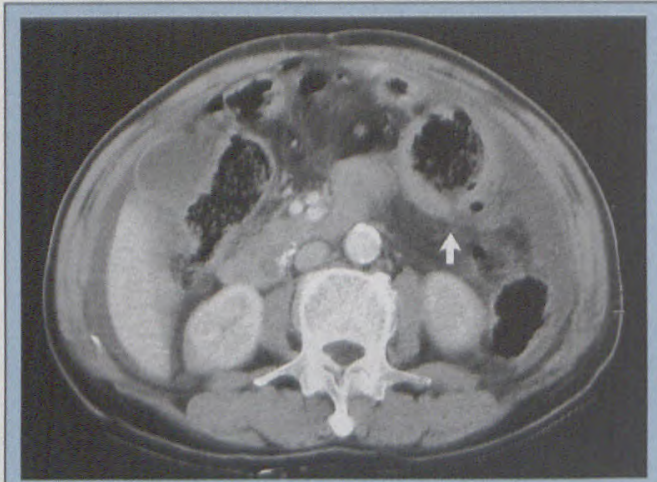

Figure 2: More caudal image directly imaging the perforation (arrow) and showing an adjacent gas bubble.

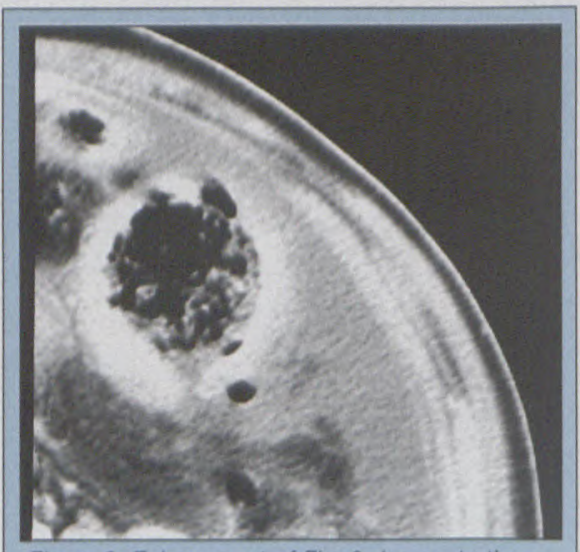

Figure 3: Enlargement of Fig. 2 demonstrating the perforation more clearly. presence of a perforated peptic ulcer measuring $15 \times 20 \mathrm{~mm}$ (mean 17.5 $\mathrm{mm}$ ) on the posterior stomach wall at the site of the gastrojejunostomy (Figure 4) as well as extensive peritonitis.

\section{Discussion}

There are several reports on the CT findings in perforated PU. These findings are of extraluminal gas and/or extraluminal oral contrast. In a retrospective study involving 35 patients with PU, two had perforation demonstrated on CT although the actual perforation itself could not be identified. ${ }^{\prime}$ Similarly, in a report of three cases of perforated PU, the site of perforation, i.e. gastric or duodenal, could be identified by the location of extraluminal gas or oral contrast, but not the actual perforation itself. ${ }^{4}$ However, Fultz et al could identify a discontinuity of the bowel wall at the site of perforation in three of nine patients with perforation due to continuity of intra- and extraluminal oral contrast through the defect. ${ }^{3}$ In our case the actual perforation itself was clearly seen even without the use of oral contrast. It is not clear why, given the incidence of perforation in PU, direct visualization of the defect does not appear to have been reported before. This perforation was no larger than normal; its size (17.5 $\mathrm{mm}$ ) was the same as the mean diameter $(17.6 \mathrm{~mm})$ reported in a series of 80 cases of perforated PU. ${ }^{5}$

Allowing for the role of non-operative management of a sealed-off perforated PU, the presence of free gas or extravasated oral contrast in the appropriate clinical setting indicates the need for operative intervention to repair the perforated viscus. Actual visualisation of the perforation itself as in this case, however, unequivocally confirms the need for urgent surgery. In

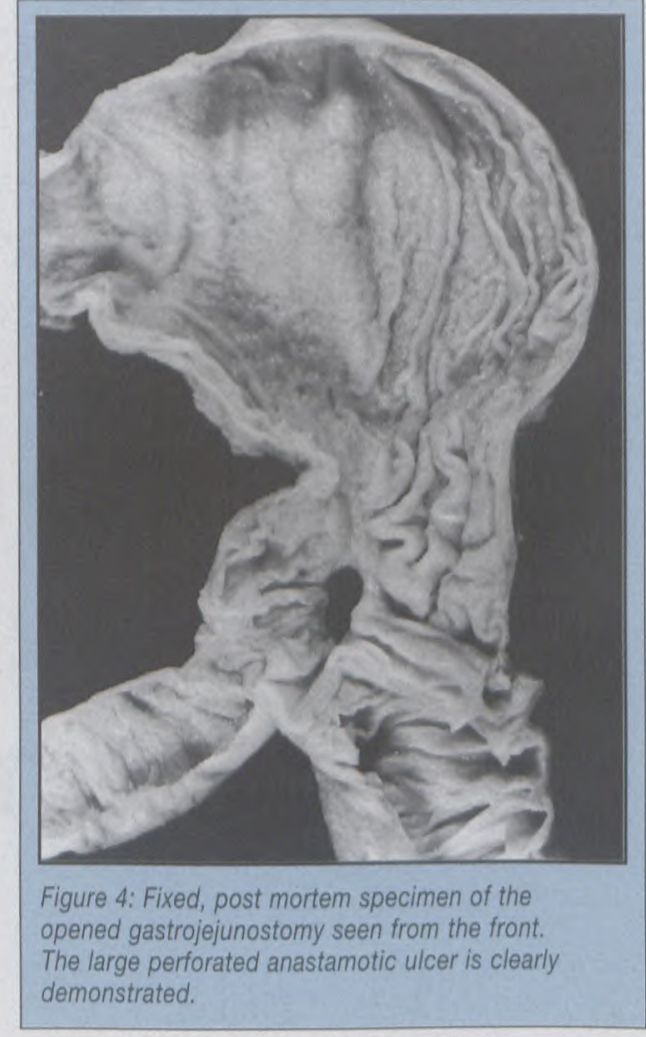

addition the knowledge of the exact site of the perforation will enable the surgeon to locate and repair it as quickly as possible. We feel that this case also suggests that in patients with an acute abdomen of uncertain cause, where perforated $\mathrm{PU}$ is suspected, there may be a role for helical scanning with reconstructions in the sagittal and coronal planes. This should increase the likelihood of directly demonstrating these often sizeable defects.

\section{References}

1. Jacobs JM, Hill MC, Steinberg WM. Peptic Ulcer Disease: CT Evaluation. Radiology 1991;178:745-748

2. Nyhus LM, Sheaff CM. Recurrent Ulcer. In: Wastell C, ed. Surgery of the esophagus, stomach and small intestine 5th ed. Boston: Little Brown, 1995:531-540

3. Fultz PJ, Skucas J, Weiss SL. CT in Upper Gastrointestinal Tract Perforations Secondary to Peptic Ulcer Disease. Gastrointest Radiol 1992;17:58

4. Jeffrey RB, Federle MP, Wall S. Value of Computed Tomography in Detecting Occult Gastrointestinal Perforation. J Comput Assist Tomog 1983; 7 (5): 825 827.

5. Horowitz J, Kukora JS, Ritchie WP. All Perforated Ulcers are not alike. Ann Surg 1989;209(6):693-697. 